\title{
Acceptability of Behavioral Treatments for Insomnia
}

\author{
Daniel Bluestein, MD, MS, CMD, AGSF, Amanda C. Healey, PhD, LPC-MHSP, NCC, \\ and Carolyn M. Rutledge, PhD, FNP-BC
}

Background: Behavioral treatments for insomnia are safe and efficacious but may not be embraced by patients in primary care. Understanding factors associated with acceptability can enhance successful use of these modalities. The objective of this study was to identify demographic and clinical/psychosocial correlates of behavioral insomnia treatment acceptability.

Methods: This nonexperimental, inventory-based, cross-sectional study enrolled patients from a hospital-sponsored primary care clinic and 2 urban academic family practices. Participants $(n=236)$ were 18 years of age or older who had clinically significant insomnia (Insomnia Severity Index score $\geq 8$ ) and were recruited consecutively at these sites. A study coordinator obtained informed consent then distributed survey materials. Participants received a $\$ 10$ honorarium. The main outcome measure was the Acceptability Insomnia Treatment Acceptability Scale-Behavioral subscale (ITAS-B).

Results: Only acceptability of medications $(r=0.259)$ and dysfunctional beliefs $(r=0.234)$ scores had significant bivariate correlations with ITAS-B scores $(P<.001)$. Medication acceptability, dysfunctional beliefs, and self-efficacy accounted for $12.45 \%$ of ITAS-B variance in linear regression.

Conclusions: Screening for dysfunctional beliefs about sleep may identify patients with interest in behavioral approaches. Improving self-efficacy for sleep may improve acceptance of behavioral insomnia therapies. Interest in behavioral and medication treatments are not mutually exclusive. However, the modest variance reported here suggests other factors impact acceptance of behavioral treatments. (J Am Board Fam Med 2011;24:272-280.)

Keywords: Insomnia, Patient Acceptance of Health Care, Primary Health Care

Primary care clinicians prioritize disorders that are common, costly, and severe. Placed in this perspective, insomnia is an important primary care concern. ${ }^{1}$ An estimated 40 to 70 million Americans are affected with insomnia intermittently, and $10 \%$ to $20 \%$ have chronic insomnia. ${ }^{2}$ Insomnia costs ex-

This article was externally peer reviewed.

Submitted 19 October 2010; revised 4 January 2011; accepted 10 January 2011.

From the Department of Family and Community Medicine, Geriatrics Division, Eastern Virginia Medical School, Norfolk (DB); the Department of Educational Leadership and Counseling, Sam Houston State University, Huntsville, TX (ACH); and the Nursing Practice Program, School of Nursing, Old Dominion Univesrity, Norfolk, VA (CMR).

Funding: American Academy of Family Physicians Foundation grant no. G0605.

Conflict of interest: none declared.

Corresponding author: Daniel Bluestein, MD, MS, CMD, AGSF, Professor and Director, Geriatrics Division, Department of Family and Community Medicine, Eastern Virginia Medical School, 825 Fairfax Avenue, Norfolk, Virginia 23507 (E-mail: bluestda@evms.edu). ceed $\$ 42$ billion each year. ${ }^{3}$ Consequences include mood disturbances, medication habituation, memory impairment, daytime fatigue, vocational and interpersonal difficulties, increased health care utilization, impaired health status, ${ }^{4-6}$ and accidents. ${ }^{7}$

Insomnia is often treated with hypnotic medications, ${ }^{8}$ Although indicated for the short term, ${ }^{9}$ medication usage may become chronic. ${ }^{10-12}$ Sedation, abuse, physical and psychological dependence, rebound insomnia, ${ }^{13}$ falls, and motor vehicle accidents $^{14,15}$ are potential sequelae. Newer agents have less abuse potential ${ }^{16}$ but are costly ( $\$ 100$ to $\$ 150$ per month) ${ }^{17,18}$ and predispose the individual to daytime sedation, psychological habituation, and accidents. ${ }^{19}$

Concerns about drug treatments have fostered interest among primary care clinicians in behavioral interventions for insomnia. Evidence-based techniques include stimulus control, relaxation, sleep restriction, paradoxical intent, sleep hygiene, 
and cognitive-behavioral therapy. ${ }^{20,21}$ Compared with medications, which are symptomatic treatments, behavioral approaches lead to sustained benefit because they address perpetuating behaviors and beliefs. ${ }^{22}$ Further advantages compared with medications include no known side effects.

Nonetheless, patients may not embrace behavioral treatments. Some may perceive receiving a behavioral treatment as evidence of having a psychiatric disorder. Such views may be associated with poverty, lack of formal education, and racial or ethnic minority status, ${ }^{23-25}$ perhaps because stigmatization of treatments for "mental illness" is more likely among these groups. ${ }^{26-29}$ Conversely, demographic predictors of acceptance include female sex and higher educational attainment. ${ }^{30}$ Effects of age are unclear; some studies show positive orientation and others negative. ${ }^{31}$

Behavioral treatments also require effort from a motivated patient. Several clinical and psychosocial variables may impact this motivation. Impaired health status has been shown to forecast drop-out from behavioral treatment programs for insomnia. ${ }^{32}$ Depression has been identified with reduced adherence and impaired response to cognitive-behavioral therapy for insomnia. ${ }^{33}$ Self-efficacy for sleep is another potential predictor because high self-efficacy predicts readiness to undertake behavioral change, whereas low self-efficacy points to a lack of readiness to take action. ${ }^{34}$ Distorted beliefs and attitudes concerning insomnia may accentuate anxiety about not sleeping, and thus patients desire rescue by medications. ${ }^{35}$ Insomnia severity has potential import as a mediator of the urgency of help-seeking. ${ }^{36,37}$

It is not known which of these demographic and clinical/psychosocial predictors have greatest salience. Understanding which are most strongly associated with acceptability, or its lack, can enhance successful use of behavioral treatments. Specifically, knowing how relationships vary across sociodemographic subgroups can direct educational interventions toward groups with lower interest. Preferences for drug therapy would suggest a need for education about risks and benefits of drugs as well as the availability of nondrug alternatives. If poor health status predicts low interest, then selfrated health or other short health status questionnaires could screen for readiness to use behavioral modalities. Depression, if predominant, can be treated before or concurrently with the introduc- tion of behavioral insomnia approaches. Motivational interviewing strategies would be indicated should low self-efficacy be a net predictor of low acceptance. Elicitation of dysfunctional beliefs and attitudes about sleep may inform educational approaches. If insomnia severity mediates acceptance, then assessment of severity could help select patients who are motivated to use behavioral or mixed treatment modalities. Accordingly, this study was undertaken to identify individual and net demographic and clinical/psychosocial correlates of behavioral insomnia treatment acceptability among primary care patients.

\section{Methods \\ Design}

This nonexperimental, inventory-based, cross-sectional study assessed relationships between the willingness to engage in behavioral intervention for the treatment of insomnia and demographic factors, acceptability of medications, health status, depression, self-efficacy for sleep, dysfunctional beliefs about sleep, and insomnia severity. Methods for this study closely parallel those detailed in a related article concerning psychosocial correlates of insomnia severity, which was previously published in The Fournal of the American Board of Family Medicine. ${ }^{38}$ Accordingly, key elements are summarized here, as are details about measures of treatment acceptability, which were not reported previously.

\section{Participants}

Participants were recruited consecutively from patients 18 years of age or older who were seen for care (any reason; not confined to seeking care for sleep problems) at 3 clinical sites. These included a hospital-sponsored primary care clinic (site 1) and 2 urban, academic family practice centers (sites 2 and 3). The study was advertised by flyers posted in waiting rooms and examination rooms. Exclusionary criteria included being younger than 18 , being illiterate, or lacking the cognitive capacity to complete informed consent or respond to surveys. Inclusion criteria entailed being age 18 or older and having clinically significant insomnia, as indicated by a score of $\geq 8$ on the Insomnia Severity Index (ISI). This is a 7-item questionnaire that asks respondents to rate severity of recent problems with sleep onset, sleep maintenance, early waking, and impact of insomnia using a 5-point Likert scale 
$(0=$ not at all, $4=$ extremely $)$. ISI scores may range from 0 to 28 . Higher scores indicate more severe insomnia, within 4 categories: absence of insomnia (0-7), mild insomnia (8-14), moderate insomnia (15-21), and severe insomnia $(22-28){ }^{39}$

\section{Data Collection}

After the approval of the study by the institutional review board, a study coordinator (ACH) obtained informed consent then distributed survey packets to participants. Surveys took between 20 to 30 minutes to complete. The coordinator was available to provide clarification if requested. Participants received a $\$ 10$ cash honorarium after completion. Surveys were then stored without names or other means of personal identification. Data were entered and stored in a secure, password-protected database accessible only to members of the research team.

\section{Measures}

Treatment preferences were measured using the Insomnia Treatment Acceptability Scale (ITAS). ${ }^{40}$ The ITAS describes 2 equally effective treatment options. The first is a nondrug treatment method aimed at teaching self-management approaches to improving sleep. The other is a new medication (no drug is named) to reduce physiologic and cognitive arousal and to reduce nighttime awakening. Respondents rate each treatment on identically worded, 8-item subscales (behavioral [ITAS-B] and medication [ITAS-M]), both of which are scored by visual analog on a $100-\mathrm{mm}$ line. Total score for each subscale is the average of the 8 items, with higher scores indicating greater willingness to utilize the treatment. Reported Cronbach's $\alpha$ values for the ITAS-B and ITAS-M range from 0.80 to 0.87 , respectively. ${ }^{41,42}$ In this study, the Cronbach's $\alpha$ values were similar, being 0.859 and 0.861 for the ITAS-B and ITAS-M, respectively. The ITAS-B is used in this study to assess the outcome variable (acceptability of behavioral sleep interventions). The ITAS-M measures acceptability of medications as a potential predictor of behavioral acceptability.

Measured sociodemographic variables included age, sex, race, marital status, and education. All were assessed by a researcher-designed survey. Age was measured as ratio (continuous) level data. Sex, race, marital status, and educational level were categorical. Health status was measured with the 8-item Short Form (SF-8) ${ }^{43}$; depressive symptoms were measured with the Center for Epidemiologic Studies-Depression scale $(\mathrm{CES}-\mathrm{D})^{44}$; self-efficacy was measured with the Self-Efficacy for Sleep scale $(\mathrm{SE}-\mathrm{S})^{45}$; dysfunctional beliefs regarding sleep were measured with the Dysfunctional Beliefs about Sleep (DBAS) scale ${ }^{46,47}$; and insomnia severity was measured with the ISI, as discussed above ${ }^{48}$ Reliability and validity of these measures is detailed in our earlier report. ${ }^{38}$

\section{Statistical Analyses}

Statistical analyses first entailed characterization of participants using descriptive and summary statistics (mean and SD for continuous variables; percentages for categorical variables). Individual associations with behavioral acceptability were examined using Pearson correlation coefficients for continuous data and Spearman correlation coefficients for ordinal data. All variables were screened for normality before conducting analysis. Means were imputed for 5 cases that had less than 2 missing values on the CES-D. Step-wise linear regression was then conducted to provide an exploratory examination regarding acceptability of medications, health status, depression, self-efficacy, dysfunctional beliefs, and insomnia severity as net predictors of acceptability of behavioral treatments.

\section{Results}

There were 236 participants: 163 from site 1, 56 from site 2 , and 17 from site 3 . As shown in Table 1 , mean age was 45 years (range, 19-91 years), with 221 participants reporting. Participants were 74\% women ( $\mathrm{n}=236), 74 \%$ African American ( $\mathrm{n}=$ $160)$, and $36 \%$ were married $(\mathrm{n}=236)$. Of 235 participants reporting educational level, $64 \%$ reported a high school education, $17 \%$ were college graduates, and $19 \%$ reported postgraduate education. Ten percent (30 participants) were age $\geq 65$ years.

To obtain a significant effect size to achieve a power level $\geq 0.80$ while detecting at least a moderate level of difference between correlated variables, an $\mathrm{N}=125$ would be necessary to achieve $\alpha=0.01 .^{49}$ This number was exceeded here, ensuring adequate power to achieve practical significance.

Table 2 reports means and SDs for the behavioral treatment acceptability and psychosocial predictors 


\begin{tabular}{|c|c|c|c|c|}
\hline & All Sites & Site 1 & Site 2 & Site 3 \\
\hline \multicolumn{5}{|l|}{ Age, years $(\mathrm{n}=236)$} \\
\hline Mean & 45 & 45 & 44 & 45 \\
\hline Range & $19-91$ & $19-83$ & $20-68$ & $22-91$ \\
\hline \multicolumn{5}{|l|}{$\operatorname{Sex}(n=236)$} \\
\hline Male & $61(26)$ & $45(28)$ & $16(29)$ & $1(6)$ \\
\hline Female & $175(74)$ & $118(72)$ & $40(71)$ & $16(94)$ \\
\hline \multicolumn{5}{|l|}{ Ethnicity $(\mathrm{n}=160)$} \\
\hline African American & $119(74)$ & $101(73)$ & $6(60)$ & $12(71)$ \\
\hline White & $34(21)$ & $26(20)$ & $3(30)$ & $5(29)$ \\
\hline Asian & $2(1)$ & $1(1)$ & $0(0)$ & $0(0)$ \\
\hline Hispanic & $1(0.5)$ & $1(1)$ & $1(10)$ & $0(0)$ \\
\hline Other & $4(2.5)$ & $4(3)$ & $0(0)$ & $0(0)$ \\
\hline \multicolumn{5}{|c|}{ Relationship status $(\mathrm{n}=236)$} \\
\hline Married & $84(36)$ & $56(34)$ & $19(34)$ & $9(52)$ \\
\hline Widowed & $17(7)$ & $11(7)$ & $5(9)$ & $1(6)$ \\
\hline Never married & $73(31)$ & $52(32)$ & $18(32)$ & $3(18)$ \\
\hline Divorced & $62(26)$ & $44(27)$ & $14(25)$ & $4(24)$ \\
\hline \multicolumn{5}{|c|}{ Level of education $(\mathrm{n}=235)$} \\
\hline Some high school & $43(18)$ & $34(21)$ & $8(14)$ & $1(6)$ \\
\hline High school graduate & $107(46)$ & $70(43)$ & $27(48)$ & $10(58)$ \\
\hline College graduate & 40 (17) & $26(16)$ & $10(18)$ & $4(24)$ \\
\hline Professional/graduate & $45(19)$ & $32(20)$ & $11(20)$ & $2(12)$ \\
\hline
\end{tabular}

Values provided as n (\%), except for Age, which is presented as number of years.

according to site. The mean for behavioral acceptability indicated above-average interest (ITAS-B mean, 0.65 ; range, $0.13-1.0$ ), as did the mean for medication acceptability (ITAS-M mean, 0.63; range, 0.04$0.98)$. Means for psychosocial predictors were midrange for health status (SF-8 mean, 24; range, 8-42); depression (CES-D mean, 22; range, 0-49); dysfunctional beliefs and attitudes about sleep (DBAS mean, 0.50; range, 0.06-0.94); self-efficacy (SE-S mean, 23; range, 9-45); and insomnia severity (ISI mean, 17; range, 8-29).
As noted in Tables 1 and 2, there is no significant variation by site with regard to sociodemographic attributes and means for the ITAS-B, ISI, SF-8, CES-D, DBAS, and SE-S. Accordingly, data were pooled for the subsequent regression analysis.

Table 3 reports individual correlation coefficients for independent variables with ITAS-B scores (behavioral treatment acceptability). Spearman coefficients were computed for categorical variables (sex, ethnicity, relationship status). Pearson coefficients were computed for interval/ratio

Table 2. Means and Standard Deviations for Behavioral Acceptability and Psychosocial Predictors

\begin{tabular}{lcccc}
\hline & Total & Site 1 & Site 2 & Site 3 \\
\hline ITAS-B & $0.65(0.18)$ & $0.65(0.20)$ & $0.67(0.18)$ & $0.66(0.16)$ \\
ITAS-M & $0.63(0.18)$ & $0.63(0.18)$ & $0.70(0.15)$ & $0.61(0.19)$ \\
SF-8 & $24(6.8)$ & $24(6.9)$ & $24(6.7)$ & $23(6.2)$ \\
CES-D & $22(11.2)$ & $22(11.2)$ & $23(11.6)$ & $24(9.8)$ \\
SE-S & $23(7.2)$ & $24(7.4)$ & $22(6.1)$ & $22(8.9)$ \\
DBAS & $0.50(0.15)$ & $0.51(0.16)$ & $0.50(0.14)$ & $0.52(0.14)$ \\
ISI & $17(5.4)$ & $16(5.4)$ & $20(4.2)$ & $17(7.2)$ \\
\hline
\end{tabular}

ITAS-B, Insomnia Treatment Acceptability Scale-Behavioral Subscale; ITAS-M, Insomnia Treatment Acceptability Scale-Medication Subscale; SF-8, Medical Outcomes Study SF-8 global health status measure; CES-D, Center for Epidemiologic Studies Depression Scale, SE-S, Self-Efficacy for Sleep Scale; DBAS, Dysfunctional Beliefs about Sleep Scale; ISI, Insomnia Severity Index. 
Table 3. Correlations with Insomnia Treatment Acceptability Scale-Behavioral Subscale Scores

\begin{tabular}{lr}
\hline Predictors & \multicolumn{1}{c}{$\mathrm{r}$} \\
\hline Demographic & \\
Sex & -0.074 \\
Age & -0.053 \\
Ethnicity & 0.005 \\
Relationship status & 0.034 \\
Education & -0.027 \\
Psychosocial & \\
ITAS-M* & 0.259 \\
SF-8 & 0.017 \\
CES-D & 0.121 \\
SE-S & 0.053 \\
DBAS* & 0.234 \\
ISI & 0.099 \\
\hline
\end{tabular}

${ }^{*} P<.001$.

ITAS-M, Insomnia Treatment Acceptability Scale-Medication Subscale; SF-8, Medical Outcomes Study SF-8 global health status measure; CES-D, Center for Epidemiologic Studies Depression Scale, SE-S, Self-Efficacy for Sleep Scale; DBAS, Dysfunctional Beliefs about Sleep Scale; ISI, Insomnia Severity Index.

variables (age, medication acceptability [ITAS-M], health status [SF-8], depression [CES-D], self-efficacy for sleep [SE-S], dysfunctional beliefs and attitudes about sleep [DBAS], and insomnia severity [ISI]). There were no significant associations between behavioral treatment acceptability and sociodemographic status. With regard to psychosocial predic- tors, acceptability of medication treatment (ITAS-M; $\mathrm{r}=0.259)$ and dysfunctional belief (DBAS; $\mathrm{r}=$ $0.234)$ scores were both significantly correlated with behavioral treatment acceptability scores $(P<$ $.001)$.

Linear regression models were examined to determine net predictors of behavioral treatment acceptability. Inclusion of demographic variables explained $0.2 \%$ of ITAS-B variance $\left(r^{2}=0.002\right)$ and was not statistically or practically significant in a step-wise regression analysis; hence these factors were not considered further. Interrelationships of psychosocial predictors required further exploration. A simple linear regression was used to determine the contribution of each psychosocial factor. Medication acceptability had the strongest bivariate association with behavioral acceptability. However, medication acceptability also was strongly related to other psychosocial predictors. Accordingly, regression models without (Table 4) and with (Table 4) medication acceptance were examined. The model excluding medication preference explained $9.3 \%$ of the variance in ITAS-B scores. In this model, both dysfunctional beliefs and self-efficacy for sleep explained strong net associations with behavioral acceptability $(P<.001)$. Insomnia severity was also significantly associated with behavioral treatment acceptability at a $P<.05$ significance level. Medication acceptance emerged as the strongest single net predictor of behavioral treatment acceptability, explaining $8.4 \%$ of variance in ITAS-B scores. When

Table 4. A: Initial Regression Analysis for Behavioral Treatment Acceptability and Regression Analysis for Behavioral Treatment Acceptability Including Medication Acceptability

\begin{tabular}{|c|c|c|c|c|c|}
\hline & $\mathrm{R}^{2}$ & $\mathrm{~F}$ & Adjusted $\mathrm{R}^{2}$ & $\beta$ & $P$ \\
\hline \multicolumn{6}{|c|}{ Initial regression analysis for BTA } \\
\hline Linear model & 0.113 & 5.833 & 0.093 & & \\
\hline Dysfunction beliefs & & & & 0.319 & $<.001$ \\
\hline SE-S & & & & 0.006 & $<.001$ \\
\hline ISI & & & & 0.005 & $<.05$ \\
\hline \multicolumn{6}{|c|}{$\begin{array}{l}\text { Regression analysis for BTA including } \\
\text { medication acceptability }\end{array}$} \\
\hline ITAS-M model & 0.088 & 22.596 & 0.084 & 0.277 & $<.001$ \\
\hline Final model & 0.135 & 12.074 & 0.124 & & \\
\hline Dysfunction beliefs & & & & 0.252 & $<.002$ \\
\hline SE-S & & & & 0.004 & $<.01$ \\
\hline ITAS-M & & & & 0.229 & $<.001$ \\
\hline
\end{tabular}

The ISI, SF-8 and CES-D were included in the analysis but were excluded from the final model because of nonsignificant contribution to overall predictive power.

BTA, behavioral treatment acceptability; ITAS-M, Insomnia Treatment Acceptability Scale-Medication Subscale; SE-S, Self-Efficacy for Sleep Scale; ISI, Insomnia Severity Index. 
medication acceptance was included as a predictor, insomnia severity was no longer a significant factor in the model, possibly because of the significant correlation between ITAS- $M$ results and insomnia severity, as measured by the ISI $(\mathrm{r}=0.249 ; P<.001)$. The combination of preference for medication, dysfunctional beliefs about sleep, and self-efficacy for sleep created a best model at $\mathrm{F}(3,235)=12.074(P<.001)$, accounting for $12.45 \%$ of the construct variance. Depression scores and health status were included in both models (Table 4) but made no significant contribution to overall predictive power.

\section{Discussion}

This study was conducted to identify demographic and clinical/psychosocial correlates of acceptance of behavioral treatments for insomnia. Our results do not reveal any demographic associations with behavioral treatment acceptance. Acceptance of medications emerged as the strongest net predictor. Insomnia severity, depressive symptoms, and concurrent poor health status were not predictive. However, the presence of high self-efficacy for sleep-inducing behavior and dysfunctional beliefs and attitudes about sleep (anxiety over the perceived consequences of poor sleep, lack of information about insomnia causes and treatment, and unrealistic expectations of treatment) were significant net predictors of behavioral acceptance.

Together, these variables accounted for $12.45 \%$ of variance in behavioral treatment acceptance. Other unmeasured variables may explain behavioral treatment acceptance, and full understanding of acceptance determinants cannot be offered at this time. Nonetheless, in psychosocial research, wherein numerous factors influence behavior, beliefs, and preferences, variance accounted for at this level is considered substantive. ${ }^{49}$

Lack of demographic associations indicates, first, that there is no significant need to target disinterested groups for education regarding the benefits of behavioral treatments. In addition, neither educational attainment nor race/ethnicity had associations with behavioral acceptance. Thus, lower education and minority status-factors associated with resistance to behavioral and psychological treatments in other contexts- did not predict resistance to (nonacceptance of) behavioral insomnia treatments. Providers should avoid stereotypic treatment decisions by assuming disinterest based on sociodemographic attributes.
The strong net relationship between behavioral and medication acceptance is perhaps a function of insomnia severity, which is highly correlated with medication preference. In other words, patients more severely impacted by their lack of sleep may be more open to both options or to the rapid relief that medications can afford. This relationship also may reflect the characteristics of patients seen in primary care medical settings, who are accustomed to medical and medication solutions for many problems. Acceptance of both behavioral treatments and medications is both surprising and informative. The historic separation of mind and body in health care can lead clinicians to view behavioral and medication modalities as opposites and that interest in one implies disinterest in the other. However, our findings indicate that patients presenting with requests for quick relief from sleeping pills, a typical primary care scenario, should not be presumed to be unaccepting of the longer-term advantages offered by behavioral treatments.

Depression and concomitant poor health status did not predict behavioral acceptance. Assessment of depression and medical/medication contributors remains a key part of insomnia assessment and diagnosis but, based on these results, should not be used to assess patient acceptance of treatment recommendations.

The finding that self-efficacy for sleep is related to interest in behavioral treatments indicates that self-efficacy should be enhanced if behavioral treatments are to be accepted and successful. Thus, self-efficacy should be examined during the course of developing an insomnia treatment plan and fostered, if necessary, using techniques such as motivational interviewing.

The relationship between behavioral treatment acceptance and dysfunctional beliefs suggests that eliciting the latter might be used to identify persons who are open to behavioral approaches. This screening can be accomplished easily in practice during the course of eliciting a history of the insomnia complaint. Alternatively, the DBAS measure used in this study could be used. A shorter, 16-item version recently has been validated. ${ }^{50}$

Several potential limitations need to be acknowledged in a discussion of these results. First, data were collected at 3 separate sites. Comparison of results by site reveals no observable differences, but unrecognized biases may have impacted results. Second, biases resulting from misunderstanding of survey items or erroneous or falsified responses may have impacted 
results. Third, incomplete reporting of ethnicity may have obscured relationships between race and insomnia severity. Nonetheless, the study sample was predominantly women and African Americans, attributes that may limit generalizability. On the other hand, this predominantly African-American sample advances understanding of treatment preferences among a group at risk for greater insomnia severity and consequences. ${ }^{51}$ Fourth, the $\$ 10$ reimbursement for survey completion may have motivated persons without insomnia and thus no true treatment preferences to inflate ISI scores to qualify. Fifth, the crosssectional design reveals associations but not cause and effect between treatment acceptance and hypothesized predictors. In addition, patients' statements of preference may not actually translate to following through with a treatment option. Finally, despite the significant predictive power of medication preference, self-efficacy, and dysfunctional beliefs and attitudes about sleep, these variables accounted for $12.45 \%$ of variance in behavioral treatment acceptance. Other unmeasured variables also are explanatory and should be examined in future research. Such variables may include anxiety or health beliefs. Quality of the relationship with the health care provider is another potential explanatory factor because satisfaction with communication predicts treatment adherence and thus greater likelihood of efficacy. ${ }^{52}$

\section{Conclusion}

This study identifies acceptance of medication treatment, self-efficacy of sleep-inducing behaviors, and the presence of dysfunctional beliefs and attitudes about sleep as predictors of acceptance of behavioral treatments for insomnia, although other unmeasured factors are also important contributors. Compared with previous studies of treatment preferences, which were conducted mainly among patients who were engaged in behavioral treatment trials, this study was conducted in primary care, to which most insomnia patients present and where there is not a priori interest in behavioral treatments. Although our results are cross-sectional and therefore preliminary, they are potentially relevant to clinicians in several respects. First, providers should not assume that patients who present with requests for quick relief using sleeping pills are disinterested in behavioral treatments. Second, assessing and facilitating self-efficacy for sleep-enhancing behavioral change potentially can improve readiness for and acceptance of behavioral insomnia therapies. Finally, screening for dysfunctional beliefs and attitudes about sleep can identify patients who have interest in behavioral approaches. Future research using prospective research designs, a broader range of explanatory variables is needed to confirm these findings and to examine relationships to satisfaction with care, improvement in sleep quality and insomnia severity, and patient-centered clinical outcomes such as wellbeing and daytime function.

\section{References}

1. NIH State-of-the-Science Conference Statement on manifestations and management of chronic insomnia in adults. NIH Consens State Sci Statements 2005; 22(2):1-30.

2. Trans-NIH Sleep Research Coordinating Committee, US Department of Health and Human Services. National Institute of Health. 2003 national sleep disorders research plan. Available at: http://www.nhlbi. nih.gov/health/prof/sleep/res_plan/sleep-rplan.pdf. Accessed March 30, 2011.

3. Buscemi N, Vandermeer B, Friesen C, et al. Manifestations and management of chronic insomnia in adults. Evid Rep Technol Assess (Summ) 2005;(125):1-10.

4. Silber MH. Clinical practice. Chronic insomnia. N Engl J Med 2005;353(8):803-10.

5. Katz DA, McHorney CA. The relationship between insomnia and health-related quality of life in patients with chronic illness. J Fam Pract 2002;51:229-35.

6. Breslau N, Roth T, Rosenthal L, et al. Sleep disturbances and psychiatric disorders: a longitudinal epidemiological study of young adults. Biol Psychiatry 1996;39:411-8.

7. Costa E, Silva JA, Chase M, Sartorius M, Roth T. Special report from a symposium held by the World Health Organization and the World Federation of Sleep Research Societies: an overview of insomnias and related disorders-recognition, epidemiology, and rational management. Sleep 1996;19:412-6.

8. Morin CM, Wooten V. Psychological and pharmacological approaches to treating insomnia. Clin Psychol Rev 1996;16:521-42.

9. Kirkwood CK. Management of insomnia. J Am Pharm Assoc (Wash) 1999;39(5):688-96.

10. Simon GE, Ludman EJ. Outcome of new benzodiazepine prescriptions to older adults in primary care. Gen Hosp Psychiatry 2006;28(5):374-8.

11. Ohayon MM, Caulet M. Psychotropic medication and insomnia complaints in two epidemiological studies. Can J Psychiatry 1996;41:457-64.

12. Holbrook AM, Crowther R, Lotter A, Cheng C, King D. Meta-analysis of benzodiazepine use in the treatment of insomnia. CMAJ 2000;162:225-33.

13. Wilson SJ, Nutt DJ. Treatment of insomnia. Psychiatry 2007;6:301-4. 
14. Morin CM, Bastien C, Guay B, Radouco-Thomas M, Leblanc J, Vallieres A. Randomized clinical trial of supervised tapering and cognitive behavior therapy to facilitate benzodiazepine discontinuation in older adults with chronic insomnia. Am J Psychiatry 2004;161:332-42.

15. Englert S, Linden M. Differences in self-reported sleep complaints in elderly persons living in the community who do or do not take sleep medication. J Clin Psychiatry 1998;59:137-44.

16. National Institute for Clinical Excellence. Guidance for the use of zaleplon, zolpidem and zopiclone for the short-term management of insomnia. Technology appraisal 77. Available at: http://www.nice.org.uk/ nicemedia/pdf/TA077fullguidance.pdf. Accessed 24 August 2010.

17. Drugstore.com, Inc. Available at: http://www. drugstore.com [homepage]. Accessed 24 August 2010 .

18. Ward CR. Ramelteon (Rozerem) for insomnia. Am Fam Physician 2006;73(8):1437.

19. Cimolai N. Zopiclone: is it a pharmacologic agent for abuse? Can Fam Physician 2007;53(12):2124-9.

20. Morgenthaler T, Kramer M, Alessi C, et al. Practice parameters for the psychological and behavioral treatment of insomnia: an update. An American Academy of Sleep Medicine report. Sleep 2006;29(11):1415-9.

21. Morin CM, Bootzin RR, Buysse DJ, Edinger JD, Espie CA, Lichstein KL. Psychological and behavioral treatment of insomnia: update of the recent evidence (1998-2004). Sleep 2006;29(11):1398-414.

22. Morin CM. Combined therapeutics for insomnia: Should our first approach be behavioral or pharmacological? Sleep Med 2006;7(Suppl 1):S15-9.

23. Nadeem E, Lange JM, Edge D, Fongwa M, Belin T, Miranda J. Does stigma keep poor young immigrant and US-born Black and Latina women from seeking mental health care? Psychiatr Serv 2007;58(12):1547-54.

24. US Department of Health and Human Services. Mental health: a report of the Surgeon General. Rockville, MD: US Department of Health and Human Services, Substance Abuse and Mental Health Services Administration, Center for Mental Health Services; 2000.

25. Sirey JA, Bruce ML, Alexopoulos GS, et al. Perceived stigma as a predictor of treatment discontinuation in young and older outpatients with depression. Am J Psychiatry 2001;158(3):479-81.

26. Cooper-Patrick L, Powe NR, Jenckes MW, Gonzales JJ, Levine DM, Ford DE. Identification of patient attitudes and preferences regarding treatment of depression. J Gen Intern Med 1997;12:431-8.

27. Sirey JA, Bruce ML, Alexapoulos GS, et al. Perceived stigma as a predictor of treatment discontinuation in young and older outpatients with depression. Am J Psychiatry 2001;75:479-81.

28. Klap R, Unroe KT, Unutzer J. Caring for mental illness in the United States: a focus on older adults. Am J Geriatr Psychiatry 2003;11(5):517-24.
29. Skultety KM. An investigation of mental health service utilization by older adults. Dissertation Abstracts International, Section B: The Sciences \& Engineering. 2004;64(10-B):5234.

30. Morin CM, Gaulier B, Barry T, Kowatch RA. Patients' acceptance of psychological and pharmacological therapies for insomnia. Sleep 1992;15(4):302-5.

31. Morin CM, Hauri PJ, Espie CA, Spielman AJ, Buysse DJ, Bootzin RR. Nonpharmacologic treatment of chronic insomnia. An American Academy of Sleep Medicine review. Sleep 1999;22(8):1134-56.

32. Morgan K, Thompson J, Dixon S, Tomeny M, Mathers N. Predicting longer-term outcomes following psychological treatment for hypnotic-dependant chronic insomnia. J Psychosom Res 2003;54(1):21-9.

33. Vincent NK, Hameed H. Relation between adherence and outcome in the group treatment of insomnia. Behav Sleep Med 2003;1(3):125-39.

34. Bouchard S, Bastien C, Morin CM. Self-efficacy and adherence to cognitive-behavioral treatment of insomnia. Behav Sleep Med 2003;1(4):187-99.

35. Azad N, Byszewski A, Sarazin FF, McLean W, Koziarz P. Hospitalized patients' preference in the treatment of insomnia: pharmacological versus non-pharmacological. Can J Clin Pharmacol 2003; 10(2):89-92.

36. Espie CA, Inglis SJ, Harvey L. Predicting clinically significant response to cognitive behavior therapy for chronic insomnia in general medical practice: analysis of outcome data at 12 months posttreatment. J Consult Clin Psychol 2001;69(1):58-66.

37. Aikens JE, Rouse ME. Help-seeking for insomnia among adult patients in primary care. J Am Board Fam Pract 2005;18(4):257-61.

38. Bluestein D, Rutledge CM, Healey AC. Psychosocial correlates of insomnia severity in primary care. J Am Board Fam Med 2010;23:204-11.

39. Morin CM, Beaulieu-Bonneau S, LeBlanc M, et al. Self-help treatment for insomnia: a randomized controlled trial. Sleep 2005;28(10):1319-27.

40. Morin CM. Insomnia: psychological assessment and management. New York: Guilford Press; 1993:104, 205-7.

41. Keenan RA, Wild MR, McArthur I, Espie CA. Children with developmental disabilities and sleep problems: parental beliefs and treatment acceptability. J Appl Res Intellect Disabil 2007;20:455-65.

42. Vincent N, Lionberg C. Treatment preference and patient satisfaction in chronic insomnia. Sleep 2001; 24(4):411-7.

43. SF-36.org. The SF-8 Health Survey. Available at: http://www.sf-36.org/tools/sf8.shtml. Accessed 24 August 2010

44. Lewinsohn P M, Seeley JR, Roberts RE, Allen NB. Center for Epidemiologic Studies Depression Scale (CES-D) as a screening instrument for depression among community-residing older adults. Psychol Aging 1997;12:277-87. 
45. Lacks P. Behavioral treatment for persistent insomnia. New York, NY: Pergamon Press; 1987:79.

46. Morin CM. Insomnia: psychological assessment and management. New York, NY: Guilford Press; 1993.

47. Morin CM, Blais F, Savard J. Are changes in beliefs and attitudes about sleep related to sleep improvements in the treatment of insomnia? Behav Res Ther 2002;40(7):741-52.

48. Bastien CH, Vallières A, Morin CM. Validation of the insomnia severity index as an outcome measure for insomnia research. Sleep Med. 2001;2:297-30.

49. Cohen J. Quantitative Methods in Psychology: A Power Primer. Psych Bulletin 1992;112(1):155-159.
50. Morin CM, Vallières A, Ivers H. Dysfunctional beliefs and attitudes about sleep (DBAS): validation of a brief version (DBAS-16). Sleep. 2007;30(11): 1547-54.

51. Mezick EJ, Matthews KA, Hall M, Strollo PJ Jr., Buysse DJ, Kamarck TW, Owens JF, Reis SE. Influence of race and socioeconomic status on sleep: Pittsburgh SleepSCORE project. Psychosom Med. 2008;70(4):410-16.

52. Epstein D. Treatment preference: applications in insomnia research: behavioral interventions for insomnia: participant preferences. Commun Nurs Res. 39:163, 2006. 